\title{
So werden sie abgerechnet
}

Dr. med. U. K., Hausärztliche Internistin, KV Thüringen:

Kann ich notwendige Impfungen jederzeit abrechnen?

Antwort: Es muss grundsätzlich zwischen Privatpatienten und gesetzlich Versicherten unterschieden werden. Für die GKV gilt die Schutzimpfungs-Richtlinie (SI-RL), die von der Ständigen Impfkommission (STIKO) erstellt wird. Zur Zeit ist es der Regelfall, dass der Gemeinsame Bundesausschuss (GB-A) die Empfehlungen der STIKO übernimmt. Damit werden die SI-RL für die Kassen verpflichtend.

Theoretisch muss die Abrechnung der Impfleistungen - der Länderhoheit folgend - in regionale Impfvereinbarungen umgesetzt werden. Dieser Bürokratismus führt häufig zu erheblichen Verzögerungen. Die Veröffentlichung geht dann zusätzlich meist im alltäglichen Papierwust unter.

Mein Rat: Halten Sie sich an die Empfehlung der STIKO und rechnen Sie entsprechend der bis dahin bestehenden Bestimmungen ab. Ein Regress mit der Begründung, dass die regionalen Impfvereinbarungen noch nicht vorlagen, ist mir nicht bekannt.

Die Gebührenordnungspositionen zur Abrechnung von Impfleistungen wurden weitgehend bundesweit vereinheitlicht: Es sind die GOP 89000 ff. Da aber immer noch länder- und kassenspezifische Unterschiede bestehen, sollte sich der Vertragsarzt bei seiner KV nach den regionalen Besonderheiten erkundigen. Daraus ergibt sich auch, dass die Leistungspositionen außerhalb des EBM sind und im Abschnitt „Vertragliche Zusatzregelungen" aufgeführt werden.
In der GOÄ ist die Situation gänzlich anders: Impfleistungen finden wir im Abschnitt $C$ "Nichtgebietsbezogene Sonderleistungen“. Dort im Teil „V. Impfungen und Testungen" finden wir die GOP 375 ff.. Diese beschreiben die Impfleistungen ohne Bezug auf eine konkrete Impfung. Mir sind auch bei Privatpatienten keine Probleme bekannt, wenn Impfleistungen im Rahmen der Empfehlungen der STIKO erbracht werden.

Grundsätzlich ist es aber nicht das Problem des impfenden Arztes, ob eine Krankenversicherung oder Beihilfestelle die erfolgte Impfung erstattet. Entscheidend ist, dass eine Impfung ordnungsgemäß nach Aufklärung und mit Zustimmung des Patienten erfolgt ist. Wichtig ist, dass in der GOÄ im Gegensatz zum EBM mit den GOP $375 \mathrm{ff}$. nur die reine Impfleistung vergütet wird. Die Beratung und die Untersuchung zum Ausschluss einer Erkrankung, die eine Kontraindikation sein könnte, können nach GOP 1 und nach den GOP 5, 6, 7 oder auch dem Ganzkörperstatus nach GOP 8 abgerechnet werden.

Der Impfstoff wird auf einem Privatrezept verordnet. Es empfiehlt sich aus Sicherheitsgründen (Kühlkette) und als Service für die Patienten, die Impfstoffe in der Praxis vorrätig zu halten.

\section{Injektion von Eisenpräparaten}

\section{Geht das als IGeL durch?}

Dr. med. A. A., Allgemeinmedizin, KVN: Patienten mit Eisenmangelanämie biete ich die venöse Injektion von Eisenpräparaten an. Kann diese Leistung als Individuelle Gesundheitsleistung (IGeL) angeboten werden?

Antwort: Eine Begründung zum Angebot einer sinnvollen Individuellen Gesundheitsleistung lässt sich hier meines Erachtens nicht finden.

Liegt ein behandlungsbedürftiger Befund vor, ist die notwendige Therapie eine Leistung der vertragsärztlichen Versorgung zu Lasten der GKV. Für die i. v.-Injektion gibt es keine eigene Leistungsposition mehr im EBM. Diese Leistung ist in die Versicherungspauschale eingerechnet worden. Vermutlich denken Sie an eine IGeL, um die Injektion doch abzurechnen.

Aber: Eine medizinisch notwendige GKVLeistung zu IGeLn, bringt Sie nicht nur in Kollision mit dem Vertragsarztrecht sondern Sie werden auch angreifbar nach dem Berufsrecht. Und: Warum sollte ein Patient in der vorab notwendigen schriftlichen Vereinbarung zur IGeL bestätigen, dass er auf eine medizinisch notwendige Behandlung zu Lasten der GKV verzichtet? 